\title{
Estimation Pre-Equilibrium Particle and Angular Distribution Spectra for the Neutrons Induce Nuclear Reactions on 90Zr Nuclei by using (FKK) Model
}

\author{
Abd alsatar Khlil Maroof ${ }^{1}$, Shfiq Shaker Shfiq², Akram Mohamed Ali ${ }^{3}$ \\ ${ }^{13}$ Department of Physics, College of Science, University of Anbar, Iraq. \\ ${ }^{2}$ Department of Physics, College of Science, University of Baghdad, Iraq, Al-Ayen University, Thi-Qar, Iarq. \\ abdalsatarkhlil@gmail.com ${ }^{1}$
}

Article History: Received: 11 October 2020; Accepted: 27 December 2020; Published online: 8 February 2021

\begin{abstract}
Particles pre - equilibrium spectra and angular distribution are calculated by usingFeshbach-Kerman- Koonin (FKK) model with PRECO-2006 code. The angular distribution of the nuclei of nuclear reactions between the nucleus of a target (90Zr) and the tapline of light particles wascalculated by using reactions of the multistep compound (MSC) and of multistep direct (MSD).Isospin, the finite well depth, and shell effects are considered. Byusing [(_40^90) 『Zr】_50 ] as target material the cross section of [90Zr(n,n)90Zr], [90Zr(n,p)90Y], [90Zr(n,D)89Y], [90Zr(n,T)88Y], [90Zr(n,3He)88Sr] and $[90 \mathrm{Zr}(\mathrm{n}, 4 \mathrm{He}) 87 \mathrm{Sr}]$ reactions were estimated. Values of cross sections estimated are 550, 371, 16.3, 4.55, 0.271 and 2.35 $\mathrm{mb} / \mathrm{MeV}$ respectively. Also the angular distribution of same nuclear reactions were estimated, and the values of angular distribution are $850,392,4.55,4.55,0.27$ and $2.35 \mathrm{mb} / \mathrm{sr} . \mathrm{MeV}$ respectively.
\end{abstract}

Keywords: Cross section, exciton, FKK model, Kalbach, preequilibrium

\section{Introduction}

Nuclear physics is that part of physics that studies nuclei's construction and interactions[1]. From this part of physics, we can see how nuclear reactions are conducted as well as a production of isotopes for various elements of the periodic table. All nuclear reaction can be represented as:

Or by the relationship :

$$
\mathrm{X}+\mathrm{x} \rightarrow \mathrm{Y}+\mathrm{y}+\mathrm{Q} \text {. }
$$

$$
\mathrm{X}(\mathrm{x}, \mathrm{y}) \mathrm{Y}
$$

Where $\mathrm{x}$ is a incident particle (projectile) that strikes target $\mathrm{X}$ and produces residual nucleus $\mathrm{Y}$ and particle $\mathrm{y}$. $\mathrm{Q}$ is the energy released or absorbed in the response, so that $\mathrm{Q}$ is positive for exoergic and negative for endoergic responses. A neutron $(\mathrm{n})$, proton $(\mathrm{p})$, deuteron $(\mathrm{d})$, triton $(\mathrm{t})$ and alpha particles or any other heavy ion may be the bombarding particle. The particles emitted can be a nucleon, nucleon, nucleon, nucleus, or gamma[2,3]. Nuclear reaction mechanisms included the direct and pre-equilibrium reactions. Many types of reactions and several forms of particulate emissions are studied and has developed in different effective models [4]. Most models deal with semi-classical approach techniques collected with statistical theory of Feshbach, Kerman, and Koonin (FKK), where the considering reactions are included: knockout, pick-up, elastic and inelastic scattering [5].

\section{The pre-equilibrium energy spectrum:}

To calculating the energy spectrum of nucleons neutrons and protons induced nuclear reactions with (90Zr) as a target nuclei, There are many mechanisms were used to this porous. The pre equilibrium mechanism, which defined Exciton model by two components and included primary and secondary nucleon emissions. the particle emission rates of type (b) as a function of

energy (E) state particular class states in the [spin mixed case given by [6]:

$$
W_{b}\left(p, p_{\pi}, E, \varepsilon\right)=\frac{2 s_{b}+1}{\pi^{2} \hbar^{3}} \mu_{b} \varepsilon \sigma_{b}\left(\varepsilon_{b}\right) \times \sum_{T_{B}}\left[C_{b}\left(T, T_{B}\right)\right]^{2} \frac{W_{e f f}\left(p_{\pi}-Z_{b,} h_{\pi}, p_{v}-N_{b,} h_{v} U, T_{B}\right.}{\omega\left(p_{\mu}, h_{v}, p_{v}, E, T\right)}(\mathbf{1})
$$

The total residual state density represented by:

$$
w_{e f f}\left(p_{\pi}-Z_{b,} h_{\pi,} p_{v}-N_{b}, h_{v}, U\right)=\sum_{i=i_{\min }}^{h_{\pi}} \sum_{j=j_{\min }}^{h_{v}} w\left(p_{\pi}-Z_{b}, i, p_{v}-N_{b}, j, U\right)(2)
$$

When can be excited by stripping the reaction [6,7], the total residual state density takes into account more compound configurations, Where $\mathrm{Cb}(\mathrm{T}, \mathrm{TB})$ in the escape channel is the Clebsch-Gordan isospin coupling vec- 
tor,TB is the quantity of isospin in the residual nucleus, $\varepsilon$ is the energy of single particles, $\mathrm{T}$ is the isospin quantum number, $\mathrm{Zb}$ is the emitted particle proton number, $\mathrm{Nb}$ is theemitted particle neutron number and $\mu \mathrm{b}$ is the reduced mass. The effectiveness of isospin in the residual nucleus, and the residual energy of excitation $\mathrm{U}=\mathrm{E}-\varepsilon-\mathrm{Bb}$, where $\mathrm{Bb}$ is the binding energy of emitted particles. The total energy range of the pre-equilibrium model in the center of the mass system for the emitted particles (b) at energy ( $\varepsilon$ ) and spin-dependent formulation is obtained by $[6,8,9]$ :

$$
\begin{aligned}
{\left[\frac{d \sigma_{a, b}(\varepsilon)}{d \varepsilon_{a}}\right]_{p r e} } & =\sum_{T}\left[\frac{d \sigma_{a, b}(\varepsilon, T)}{d \varepsilon_{a}}\right]_{p r e} \\
& =\sigma_{a}\left(\varepsilon_{a}\right) \sum_{T}\left|C_{a}\left(T, T_{A}\right)\right|^{2} \times \sum_{p} \sum_{p_{\pi}} S_{p r e}\left(p, p_{\pi}, T\right) W_{b}\left(p, p_{\pi}, E, \varepsilon, T\right)
\end{aligned}
$$

Where $\sigma_{\text {a.pre }} \square$ is the cross section for modeling the complex nucleus that reduced by a cross section with direct reaction and $(\mathrm{P}, \mathrm{p} \pi, \mathrm{T})$ is the average amount of time spent in each class of configuration [7].For each spin, $\mathrm{T}$, the product of equation (3) is added and multiplied by the Clebsch-Gordan coefficient of coupling of the entry channel isospin.Since a second particle emission can occur at the pre-equilibrium point, then the emission may influence the energy spectra at high energy excitation [10]. Within the FKK model the multi-step direct (MSD) or pre-equilibrium or forward-peaked component involves the pre-equilibrium exciton process components. As well as knockout and inelastic scattering, cross-sections of nucleon, (IN) involving cluster degrees of freedom, could be represented with:

$$
\left\lfloor d \sigma_{\varepsilon_{b}}\right\rfloor_{M S D}=\left\lfloor d \sigma_{\varepsilon_{b}}\right\rfloor_{p r e, 1}+\left\lfloor d \sigma_{\varepsilon_{b}}\right\rfloor_{p r e, 2}+\left\lfloor d \sigma_{\varepsilon_{b}}\right\rfloor_{N T}+\left\lfloor d \sigma_{\varepsilon_{b}}\right\rfloor_{I N}
$$

and for other reaction channel knockout $(\mathrm{KO})$ is,

$$
\left\lfloor d \sigma_{\varepsilon_{b}}\right\rfloor_{M S D}=\left\lfloor d \sigma_{\varepsilon_{b}}\right\rfloor_{p r e, 1}+\left\lfloor d \sigma_{\varepsilon_{b}}\right\rfloor_{p r e, 2}+\left\lfloor d \sigma_{\varepsilon_{b}}\right\rfloor_{N T}+\left\lfloor d \sigma_{\varepsilon_{b}}\right\rfloor_{K O}
$$

Where $\mathrm{KO}$ only makes a contribution for reactions $(\mathrm{N}, \alpha),(\mathrm{C}, \mathrm{N})$ and $(\mathrm{C}, \alpha)$, whereas $\mathrm{N}$ is a nucleon, $\mathrm{C}$ is a complex particle (2D, $3 \mathrm{~T}, 3 \mathrm{He}$, or $\alpha$-particle). Contains the corresponding equilibrium or symmetric element only the primary and secondary evaporation cross sections and is given the Multi stepcompound spectrum,

$$
\left\lfloor d \sigma_{\varepsilon_{b}}\right\rfloor_{M S C}=\left\lfloor d \sigma_{\varepsilon_{b}}\right\rfloor_{e q, 1}+\left\lfloor d \sigma_{\varepsilon_{b}}\right\rfloor_{e q, 2}
$$

For $\left[{ }_{40}^{90} \mathbf{Z r}_{\mathbf{5 0}}\right]$ nuclei the binding energies were initially calculated for all light nuclei from the primary emission process when the nucleus is in the excition state through a special program the connecting energies were then used during the data entry into the PRECO- 6 code and the emission spectra and angular distribution were then obtained for each particle is emitted. Different mechanisms have been used to calculate the total energy spectrum and angular distribution in terms of MSD and MSC models for the emission nucleons and light nucleiat incident (excitation) energy $5 \mathrm{MeV}$ from reactions.

[90Zr(n,n)90Zr], [90Zr(n,p)90Y], [90Zr(n,D)89Y], [90Zr(n,T)88Y], [90Zr(n,3He)88Sr] and [90Zr(n,4He)87 Sr]. In this work, many correction parameters was used to calculate the spectrum at varying incident energies. The two-component particle-hole state follow-up on transition estimates rates and then demand differentials full spectrum. Not even pre-equilibrium emissions covered by the calculations, but the particles too emissions in a State of control it considered the standardization below factors for components in the matrix $[5,11]$.

$$
\left.M_{i j}\right|^{2}=K_{i j} A_{a} g^{-3}\left(\frac{E}{3 A_{a}}+20.9\right)^{-3}(7)
$$

The angular distribution using FKK model can be listed as [11]: 


$$
\frac{d^{2} \sigma}{d \Omega d \varepsilon_{h}}=\frac{1}{4 \pi} \frac{d \sigma}{d \varepsilon_{b}} \frac{a_{e x}}{\sinh \left(a_{e x}\right)}\left[\cosh \left(a_{e x} \cos \theta\right)+f_{m s d} \sinh \left(a_{e x} \cos \theta\right)(\mathbf{8})\right.
$$

where $\boldsymbol{a e x}$ represents the slop parameter included in exciton model and its components are related. The angle $\theta$ is adjusted as center of mass measured unit [13] and the amount $\boldsymbol{f}$ msd is the fraction of energy in the FKK model at the specified emission cross section. Such cross sections are combined to find the fraction of the cross section $\boldsymbol{f} \boldsymbol{m s} \boldsymbol{d}$ which represents the pre-equilibrium part [11]:

$$
f_{m s d}(\varepsilon)=\frac{\left[d \sigma_{\varepsilon b}\right]_{m s d}}{\left[d \sigma_{\varepsilon b}\right]_{m s d}+\left[d \sigma_{\varepsilon b}\right]_{m s c}}(\mathbf{9})
$$

$\left[d \sigma_{\varepsilon b}\right]_{m s d}$ represents the pre-equilibrium emission spectra for both primary and secondary as well as the cross section from nucleon transfer, knockout and inelastic scattering involving cluster degrees of freedom. The quantity $\left[\mathrm{d} \sigma_{\varepsilon b}\right]_{\mathrm{msc}}$ is the equilibrium emission spectra or the symmetric component comprises only the crosssections of primary and secondary evaporation.

\section{Secondary emission:}

Secondary emissions are only considered for nucleons and only after a nucleon's primary emission, while primary particle emissions by mass four (alpha-particles) are still measured. Secondary emission is a weak, even following primary nucleon emission because of primary pre-equilibrium emission of complex particles is generally weaker than that of nucleons, most of their cross section comes from other processes, such as direct transfer of nucleons. Kalbach was constructed PRECO code by computational framework to calculate different parameters such as cross section from pre-equilibrium emission spectrum with angular distribution and she make capable $t$ it to estimates the contribution of secondary emissions and its effects to the emission spectrum[5]. In the present calculations, secondary emissions were only considered for nucleons when they were emitted after a primary nucleon emission but if the primary emission is a complicated particle, the secondary emission is not considered because it is likely to be soft and can be ignored.

\section{The well depth effect:}

In limiting the excitation energy that can be produced by a hole degree of freedom, the results of the finite depth $\mathrm{V}$ of the potential well. Relative to Fermi level, the well depth is determined, when all interactions occur inside the nucleus's main volume. In PRECO-2006 the full well depth is taken to be V $=\mathrm{V} 0=38 \mathrm{MeV}$ by default. In the main reaction calculations,there is evidence that near the nuclear surface, the initial contact between a projectile and a target nucleon is often localized.

\section{Results and Discussions:}

The nuclear model calculations carried out for number of reactions as $\left[{ }^{90} \mathrm{Zr}(\mathrm{n}, \mathrm{n}){ }^{90} \mathrm{Zr}\right],\left[{ }^{90} \mathrm{Zr}(\mathrm{n}, \mathrm{p}){ }^{90} \mathrm{Y}\right]$, $\left[{ }^{90} \mathrm{Zr}(\mathrm{n}, \mathrm{D}){ }^{89} \mathrm{Y}\right],\left[{ }^{90} \mathrm{Zr}(\mathrm{n}, \mathrm{T}){ }^{88} \mathrm{Y}\right],\left[{ }^{90} \mathrm{Zr}\left(\mathrm{n},{ }^{3} \mathrm{He}\right){ }^{88} \mathrm{Sr}\right],\left[{ }^{90} \mathrm{Zr}\left(\mathrm{n},{ }^{4} \mathrm{He}\right){ }^{87} \mathrm{Sr}\right]$ at incident neutrons $(5,25$ and 50 $\mathrm{MeV}$ ).In this side the cross-section and differential cross-section are calculated for the all previousreactions as follows.
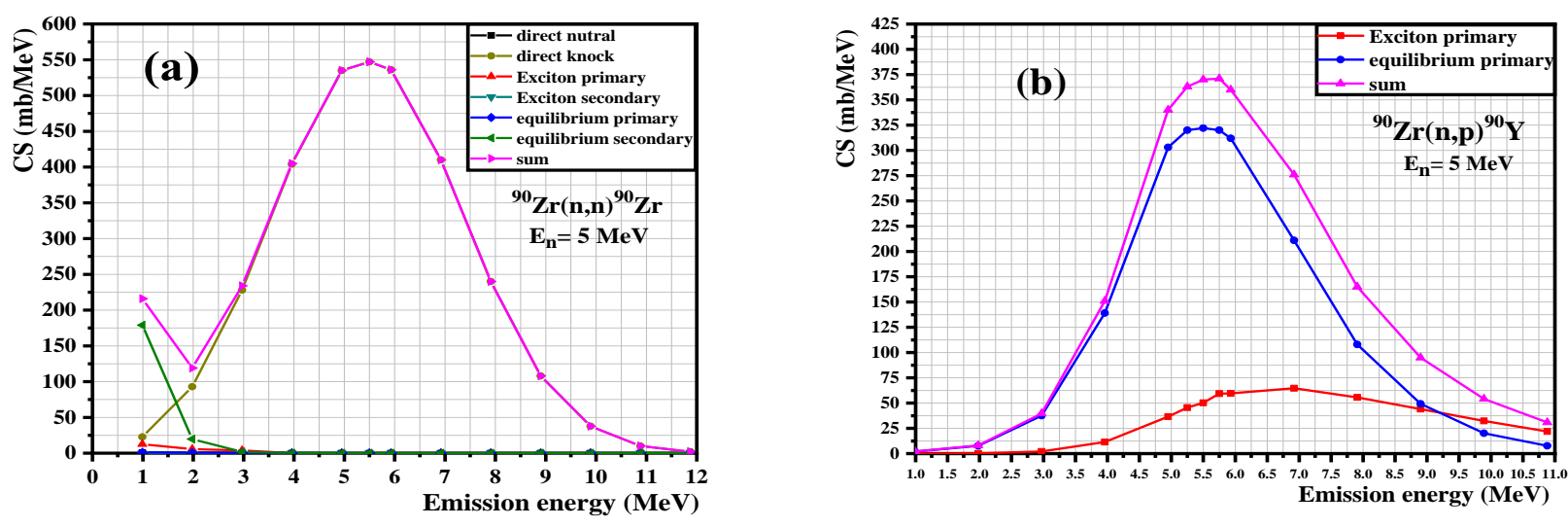

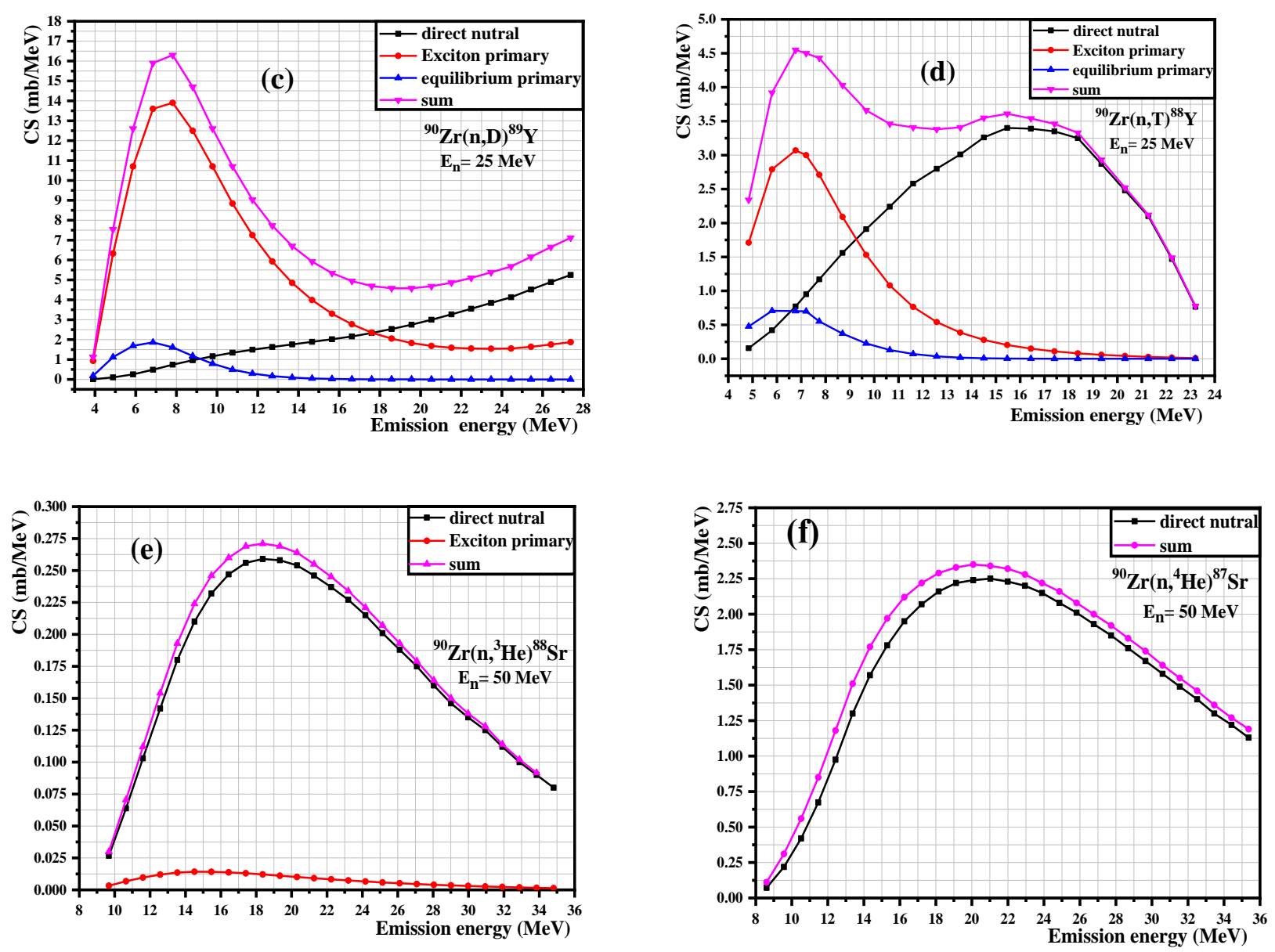

Fig(1):Particle spectra (cross section) for ${ }_{40}^{90} \mathrm{Zr}_{50}$ (a,b,c,d,e and f ) for $\quad\left[{ }^{90} \mathrm{Zr}(\mathrm{n}, \mathrm{n}){ }^{90} \mathrm{Zr}\right],\left[{ }^{90} \mathrm{Zr}(\mathrm{n}, \mathrm{p}){ }^{90} \mathrm{Y}\right]$, $\left[{ }^{90} \mathrm{Zr}(\mathrm{n}, \mathrm{D}){ }^{89} \mathrm{Y}\right],\left[{ }^{90} \mathrm{Zr}(\mathrm{n}, \mathrm{T}){ }^{88} \mathrm{Y}\right],\left[{ }^{90} \mathrm{Zr}\left(\mathrm{n},{ }^{3} \mathrm{He}\right){ }^{88} \mathrm{Sr}\right]$ and $\left[{ }^{90} \mathrm{Zr}\left(\mathrm{n},{ }^{4} \mathrm{He}\right){ }^{87} \mathrm{Sr}\right]$ respectively at different incident neutron energies .

Fig (1) show the cross section or particle spectra for some light particles which emission from [90Zr(n,n)90Zr], [90Zr(n,p)90Y], [90Zr(n,D)89Y], [90Zr(n,T)88Y], [90Zr(n,3He)88Sr] and [90Zr(n,4He)87Sr]. Fig(1.a) represent the $[90 \mathrm{Zr}(\mathrm{n}, \mathrm{n}) 90 \mathrm{Zr}]$ nuclear reaction, the results showed for a large range of emission energies, there is a peak of cross section with value $(550 \mathrm{mb} / \mathrm{MeV})$ at emission energy $(5.5 \mathrm{MeV})$, this peak is the total contributions of the direct knock component which represent thelargest contribution for cross section while the rest of the contributions as direct nutral ,exciton (primary, secondary) and equilibrium (primary, secondary) are almost very small close to zero and can be abandoned. Fig(2.a) represent the [90Zr(n,p)90Y] nuclear reaction, the results showed the emission spectra have been peaks of cross section at emission energy (5.75 $\mathrm{MeV})$, the sum contribution is the largest with value equal to $(371 \mathrm{mb} / \mathrm{MeV})$ which comes in exciton primary contribution(59.3 mb/MeV), and equilibrium primary contribution $(320 \mathrm{mb} / \mathrm{MeV})$, while the rest contribution is very small have been neglected. Fig(1.c) represent the[90Zr(n,D)89Y]nuclear reaction, the results showed the cross section was calculated by using preco-2006 code at incident neutron energy ( $25 \mathrm{MeV}$ ), the emission spectra have been peaks of cross section at emission energy $(7.82 \mathrm{MeV})$, the clear contributions comes from direct nutral contribution $(0.738 \mathrm{mb} / \mathrm{MeV})$,exciton primary contribution $(13.9 \mathrm{mb} / \mathrm{MeV})$, equilibrium primary $(1.61 \mathrm{mb} / \mathrm{MeV})$, and the total contributions $(16.3 \mathrm{mb} / \mathrm{MeV})$, while the rest contributions was very small have been neglected. Fig(1.d) represent the $[90 \mathrm{Zr}(\mathrm{n}, \mathrm{T}) 88 \mathrm{Y}]$ nuclear reaction,the cross section of this reaction was calculated by using preco-2006 code at incident neutron energy (25 MeV), the emission spectra have been peaks of cross section at emission energy $(6.77 \mathrm{MeV})$, the clear contributions comes from direct nutral contribution $(0.772 \mathrm{mb} / \mathrm{MeV})$, exciton primary contribution $(3.07 \mathrm{mb} / \mathrm{MeV})$, equilibrium primary $(0.703 \mathrm{mb} / \mathrm{MeV})$, and the total contributions $(4.55 \mathrm{mb} / \mathrm{MeV})$, while the rest contributions was very small have been neglected, we notice that there is another peak at emission energy $(15.47 \mathrm{MeV})$ but a smaller than the first peak, this appear in sum contribution with value $(3.61 \mathrm{mb} / \mathrm{MeV})$. Fig(1.e) represent the $[90 \mathrm{Zr}(\mathrm{n}, 3 \mathrm{He}) 88 \mathrm{Sr}$ ] nuclear reaction, the results at incident neutron energy $(50 \mathrm{MeV})$ showed for a large range of emission energies, there is apeak of cross section 
with value $(0.259 \mathrm{mb} / \mathrm{MeV})$ for direct nutral contribution, exciton primary contribution $(0.0121 \mathrm{mb} / \mathrm{MeV})$, and the total contributions $(0.271 \mathrm{mb} / \mathrm{MeV})$, while the rest contributions was very small have been neglected. Fig(1.f) represent the $[90 \mathrm{Zr}(\mathrm{n}, 4 \mathrm{He}) 87 \mathrm{Sr}]$ nuclear reaction, the emission spectra have been a peak of cross section at emission energy (20.08MeV), the clear contributions comes from direct nutral contribution $(2.24 \mathrm{mb} / \mathrm{MeV})$, direct knock contribution $(0.06 \mathrm{mb} / \mathrm{MeV})$, exciton primary contribution $(0.0583 \mathrm{mb} / \mathrm{MeV})$, equilibrium primary $(0.005 \mathrm{mb} / \mathrm{MeV})$, and the total contributions $(2.35 \mathrm{mb} / \mathrm{MeV})$, while the rest contributions was very small have been neglected. From the previous results we can conclude that the best cross section at $[90 \mathrm{Zr}(\mathrm{n}, \mathrm{n}) 90 \mathrm{Zr}]$ with value of cross section equal to $(550 \mathrm{mb} / \mathrm{MeV})$ comparing with the other nuclear reactions, it is due to neutrons are neutral particles approach the target nuclei experiencing no Coulomb repulsion but the particles such as alpha, protons and light nuclides with positive charge experience a repulsion of the atomic nucleus due to the electromagnetic force.

Also for the angular distribution, the nuclear model calculations carried out for number of reactions as [90Zr(n,n)90Zr], [90Zr(n,p)90Y], [90Zr(n,D)89Y], [90Zr(n,T)88Y], [90Zr(n,3He)88Sr], [90Zr(n,4He)87Sr].In this side they are angular distribution calculated for the all previousreactionsby using preco-2006 code at incident neutrons $(5,25$ and $50 \mathrm{MeV})$ and at five angles there is $(0,30,60,90,120$ and total of contributions) as follow.
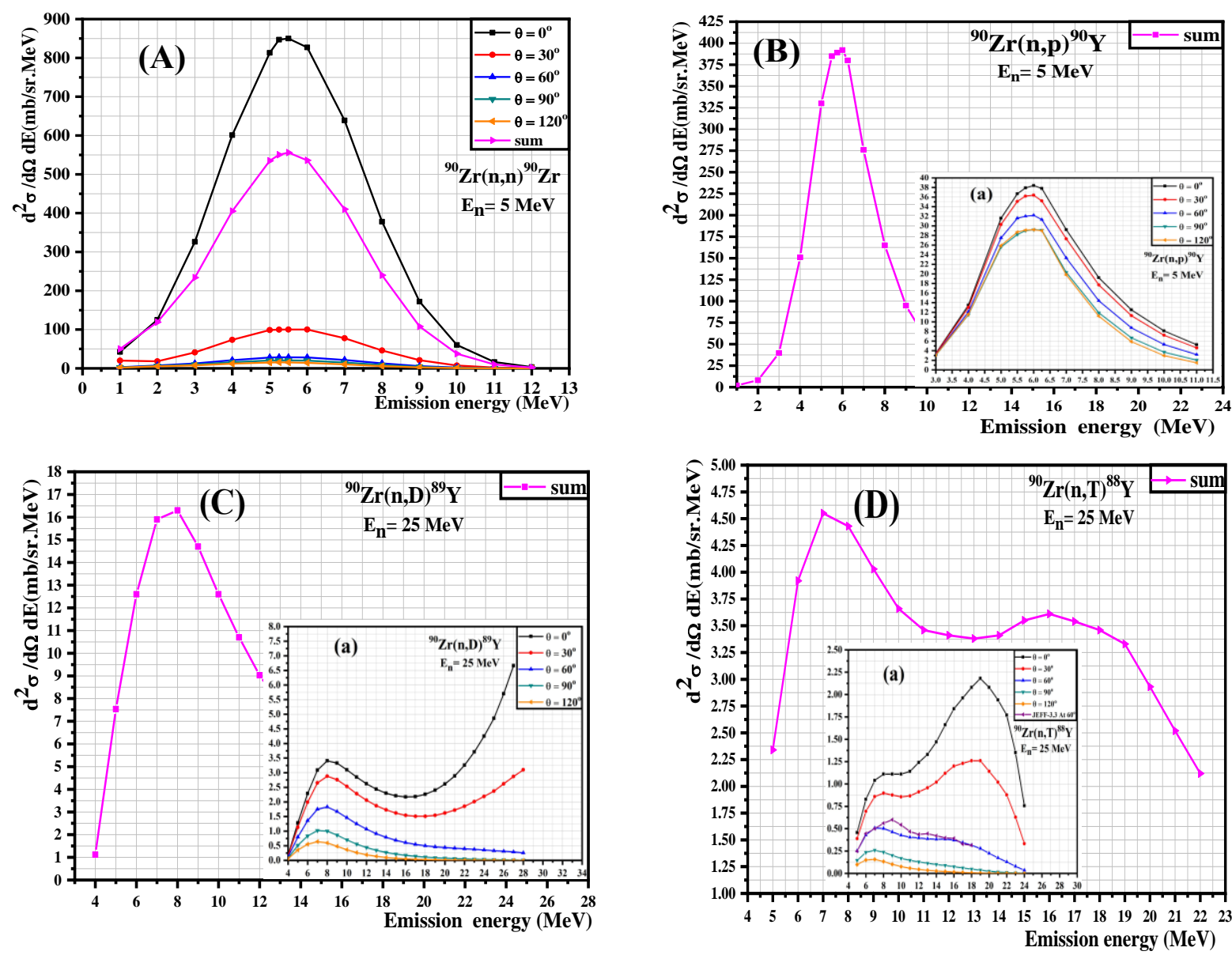

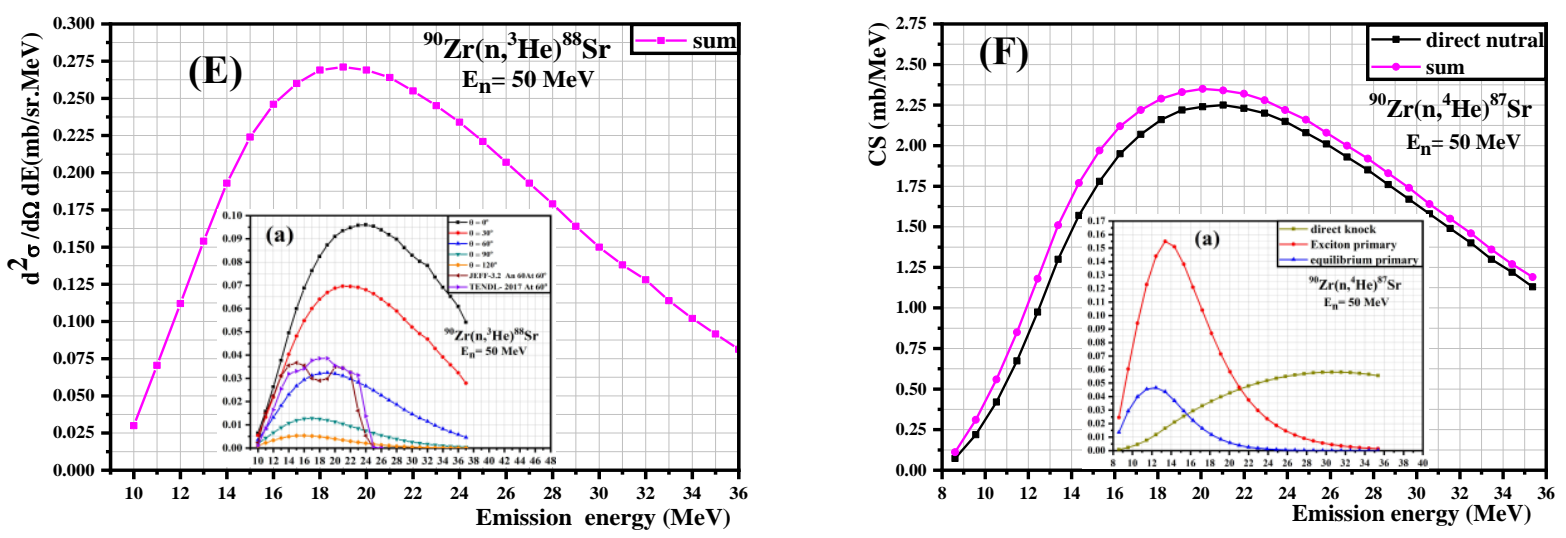

Fig(2): Angular distribution for ${ }_{40}^{90} \mathrm{Zr}{ }_{50}$ (A,B,C,D,E and F ) for $\left[{ }^{90} \mathrm{Zr}(\mathrm{n}, \mathrm{n}){ }^{90} \mathrm{Zr}\right],\left[{ }^{90} \mathrm{Zr}(\mathrm{n}, \mathrm{p}){ }^{90} \mathrm{Y}\right],\left[{ }^{90} \mathrm{Zr}(\mathrm{n}, \mathrm{D}){ }^{89} \mathrm{Y}\right]$, $\left[{ }^{90} \mathrm{Zr}(\mathrm{n}, \mathrm{T}){ }^{88} \mathrm{Y}\right],\left[{ }^{90} \mathrm{Zr}\left(\mathrm{n},{ }^{3} \mathrm{He}\right){ }^{88} \mathrm{Sr}\right]$ and $\left[{ }^{90} \mathrm{Zr}\left(\mathrm{n},{ }^{4} \mathrm{He}\right){ }^{87} \mathrm{Sr}\right]$ respectively at different incident neutron energies

Fig (2). show the angular distribution for some light particles which emission from [ $90 \mathrm{Zr}(\mathrm{n}, \mathrm{n}) 90 \mathrm{Zr}$, [90Zr(n,p)90Y], [90Zr(n,D)89Y], [90Zr(n,T)88Y], [90Zr(n,3He)88Sr] and[90Zr(n,4He)87Sr].

Fig(2.A) represent the angular distribution for[90Zr(n,n)90Zr] nuclear reaction, the result showed at incident neutron energy $(5 \mathrm{MeV})$ and at five angles there is $(0,30,60,90,120$ and total of contributions) a large range of emission energies, there are a peaks of differential cross section at some of emission energies. We notice that the largest differential cross $\operatorname{section}(850 \mathrm{mb} / \mathrm{sr} . \mathrm{MeV})$ for $\left(\theta=0^{\circ}\right)$ contribution at emission energy $(5.5 \mathrm{MeV})$, but it is equal to $(100 \mathrm{mb} / \mathrm{sr} . \mathrm{MeV})$ for $\left(\theta=30^{\circ}\right)$ contribution at emission energy $(5.5 \mathrm{MeV})$, and equal to $(556 \mathrm{mb} / \mathrm{sr} . \mathrm{MeV})$ for (sum) contribution, while there are small values of differential cross section $\left(28 \mathrm{mb} / \mathrm{sr}\right.$.MeV) at $\left(\theta=60^{\circ}\right)$ contribution, and $(20.49 \mathrm{mb} / \mathrm{sr} . \mathrm{MeV})$ at $\left(\theta=90^{\circ}\right)$ contribution, and $(15.9 \mathrm{mb} / \mathrm{sr} . \mathrm{MeV})$ at $\left(\theta=120^{\circ}\right)$ contribution. $\mathrm{Fig}(2 . \mathrm{B})$ represent the angular distribution for[90Zr(n,p)90Y] nuclear reaction was calculated by using preco2006 code at incident neutron energy $(5 \mathrm{MeV})$ and at five angles there is $(0,30,60,90,120$ and total of contributions), the total contributions which named sum is the largest value of differential cross section equal to (392 $\mathrm{mb} / \mathrm{sr} . \mathrm{MeV})$ at emission energy $(6 \mathrm{MeV})$, this contribution comes from the rest contributions, $\left(\theta=0^{\circ}\right)$ with $(38.4$ $\mathrm{mb} / \mathrm{sr} . \mathrm{MeV}), \quad\left(\theta=30^{\circ}\right) \quad$ with $(36.4 \mathrm{mb} / \mathrm{sr} . \mathrm{MeV}), \quad\left(\theta=60^{\circ}\right) \quad$ with $\quad(32.2 \mathrm{mb} / \mathrm{sr} . \mathrm{MeV}), \quad\left(\theta=90^{\circ}\right), \quad$ with $\quad(29.2$ $\mathrm{mb} / \mathrm{sr} . \mathrm{MeV})$, and $\left(\theta=120^{\circ}\right)$ with $(29.2 \mathrm{mb} / \mathrm{sr} . \mathrm{MeV})$. Fig(2.C) represent the angular distribution for[90Zr(n,D)89Y] nuclear reaction at incident neutron energy $(25 \mathrm{MeV})$ and at five angles there is $(0,30,60,90,120$ and total of contributions). The result showed that the largest value of differential cross section equal to $(4.55 \mathrm{mb} / \mathrm{sr} . \mathrm{MeV})$ at emission energy $(8 \mathrm{MeV})$, this contribution comes from the rest contribution $\left(\theta=0^{\circ}\right)$ with $(3.41 \mathrm{mb} / \mathrm{sr} . \mathrm{MeV}),(\theta$

$\left.=30^{\circ}\right)$ with $(2.88 \mathrm{mb} / \mathrm{sr} \cdot \mathrm{MeV}),\left(\theta=60^{\circ}\right)$ with $(1.83 \mathrm{mb} / \mathrm{sr} \cdot \mathrm{MeV}),\left(\theta=90^{\circ}\right)$ with $(1 \mathrm{mb} / \mathrm{sr} . \mathrm{MeV})$ and $\left(\theta=120^{\circ}\right)$ with $(0.59 \mathrm{mb} / \mathrm{sr} . \mathrm{MeV})$. Fig(2.D) represent the angular distribution for[90Zr(n,T)88Y] nuclear reaction at incident neutron energy $(25 \mathrm{MeV})$ and at five angles there is $(0,30,60,90,120$ and total of contributions). The total contributions is the largest value of differential cross section equal to $(4.55 \mathrm{mb} / \mathrm{sr} . \mathrm{MeV})$ at emission energy $(7 \mathrm{MeV})$, this contribution comes from the rest contributions $\left(\theta=0^{\circ}\right)$ with $(1.04 \mathrm{mb} / \mathrm{sr} . \mathrm{MeV}),\left(\theta=30^{\circ}\right)$ with $(0.859 \mathrm{mb} / \mathrm{sr} . \mathrm{MeV}),(\theta=$

$\left.60^{\circ}\right)$, with $(0.52 \mathrm{mb} / \mathrm{sr} \cdot \mathrm{MeV}),\left(\theta=90^{\circ}\right)$ with $(0.528 \mathrm{mb} / \mathrm{sr} \cdot \mathrm{MeV})$, and $\left(\theta=120^{\circ}\right)$ with $(0.156 \mathrm{mb} / \mathrm{sr} \cdot \mathrm{MeV}) . \mathrm{Fig}(2 . \mathrm{E})$ represent the angular distribution for $[90 \mathrm{Zr}(\mathrm{n}, 3 \mathrm{He}) 88 \mathrm{Sr}]$ nuclear reaction at incident neutron energy $(50 \mathrm{MeV})$ and at five angles there is $(0,30,60,90,120$ and total of contributions). The largest value of differential cross section equal to $(0.27 \mathrm{mb} / \mathrm{sr} . \mathrm{MeV})$ at emission energy $(19 \mathrm{MeV})$, this contribution comes from the rest contributions $\left(\theta=0^{\circ}\right)$ with $(0.087 \mathrm{mb} / \mathrm{sr} . \mathrm{MeV}),\left(\theta=30^{\circ}\right)$ with value $(0.067 \mathrm{mb} / \mathrm{sr} . \mathrm{MeV}),\left(\theta=60^{\circ}\right)$ with $(0.0324 \mathrm{mb} / \mathrm{sr} . \mathrm{MeV})$, $\left(\theta=90^{\circ}\right)$ with $(0.012 \mathrm{mb} / \mathrm{sr} . \mathrm{MeV})$ and $\left(\theta=120^{\circ}\right)$ with $(0.005 \mathrm{mb} / \mathrm{sr} . \mathrm{MeV})$. Fig(2.E) represent the angular distribution for $[90 \mathrm{Zr}(\mathrm{n}, 4 \mathrm{He}) 87 \mathrm{Sr}]$ nuclear reaction at incident neutron energy $(50 \mathrm{MeV})$ and at five angles there is $(0,30,60,90,120$ and total of contributions). The largest value of differential cross section equal to $(0.27$ $\mathrm{mb} / \mathrm{sr} . \mathrm{MeV})$ at emission energy $(19 \mathrm{MeV})$, this contribution comes from the rest contributions $\left(\theta=0^{\circ}\right)$ with $(0.522 \mathrm{mb} / \mathrm{sr} . \mathrm{MeV}),\left(\theta=30^{\circ}\right)$ with $(0.439 \mathrm{mb} / \mathrm{sr} . \mathrm{MeV}),\left(\theta=60^{\circ}\right)$ with $(0.25 \mathrm{mb} / \mathrm{sr} . \mathrm{MeV})$

$\left(\theta=90^{\circ}\right)$ with $(0.144 \mathrm{mb} / \mathrm{sr} . \mathrm{MeV})$ and $\left(\theta=120^{\circ}\right)$ with $(0.0755 \mathrm{mb} / \mathrm{sr} . \mathrm{MeV})$. From the previous results we can conclude that the best angular distribution at $[90 \mathrm{Zr}(\mathrm{n}, \mathrm{n}) 90 \mathrm{Zr}]$ with value of cross section equal to (850 $\mathrm{mb} / \mathrm{sr} . \mathrm{MeV}$ ) comparing with the other nuclear reactions, it is due to neutrons are neutral particles approach the target nuclei experiencing no Coulomb repulsion but the particles such as alpha, protons and light nuclides with positive charge experience a repulsion of the atomic nucleus due to the electromagnetic force.

\section{Isospin calculation:}

Measurements of isospin canbe carried out assuming that the isospin quantum numberis either completely mixed or fully retained during the preequilibrium part of the calculations. If the isospin is conserved during the 
pre-equilibrium part, as an input, the amount of mixing to be assumed at equilibrium is also required.State densities are needed for isospin-conserved calculations for particle-hole configurations with good isospin. In this work, the nuclear reaction [ $90 \mathrm{Zr}(\mathrm{n}, \mathrm{n}) 90 \mathrm{Zr}$ ] was studied by PRECO-2006 code and the effect of adding the Isospin conservation into the nuclear level densities on the energy spectra and for three values isospin (It=0,1 and 2 ) at incident neutron energy $(50 \mathrm{MeV})$. Those results showed no significance variation for conserved and mixed isospin calculations for all reactions as shown in the fig(3).
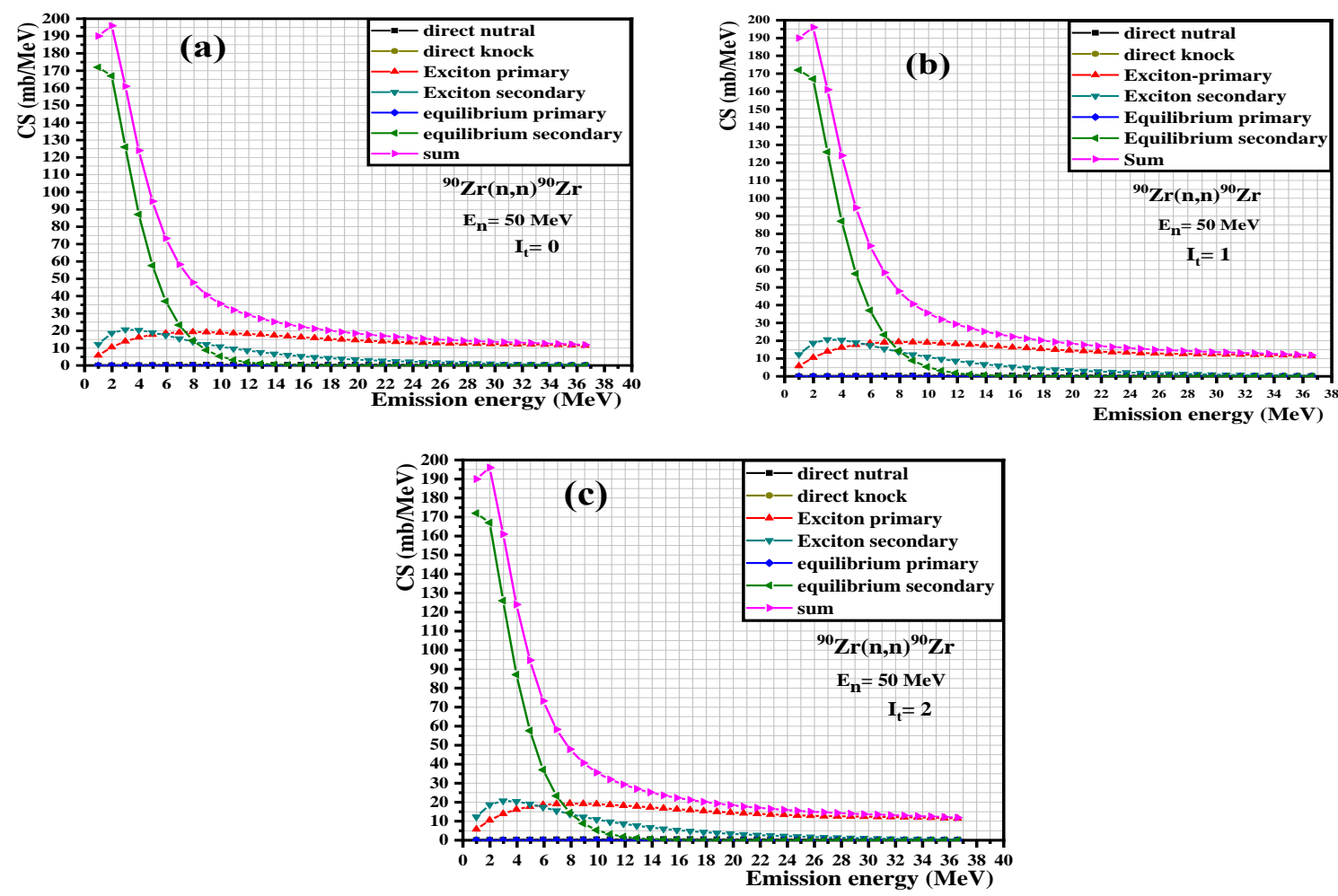

$\operatorname{Fig}[3(\mathbf{a}, \mathbf{b}, \mathbf{c})]$.Cross section for different values of isospin $I_{t}=0,1$ and 2 for ${ }^{90} \mathrm{Zr}(\mathrm{n}, \mathrm{n})^{90} \mathrm{Zr}$ reaction.

\section{The well depth calculation:}

In this work we take some values of V0 greater than $38 \mathrm{MeV}$ and less than $38 \mathrm{MeV}(\mathrm{V} 0=28$ and $48 \mathrm{MeV})$ for the nuclear reaction $[90 \mathrm{Zr}(\mathrm{n}, \mathrm{n}) 90 \mathrm{Zr}]$ which have been a value of cross section equal to $(547 \mathrm{mb} / \mathrm{MeV})$ for sum contribution at the default value of $(\mathrm{V} 0=38 \mathrm{MeV})$. We studied by PRECO-2006 codethe effect of changing the central well depth on the cross section of this nuclear reaction. The results showed that there was a clear change in the cross-section values for the nuclear reaction, this changes can be notice when the central well depth was (28 MeV), the cross section value is $(540 \mathrm{mb} / \mathrm{MeV})$ for sum contribution at emission energy $(5.5 \mathrm{MeV})$, and equal to $(560 \mathrm{mb} / \mathrm{MeV})$ when the central well depth was $(48 \mathrm{MeV})$ for the same emission energy.From these results we found the cross section increases by increments values of the central well depth. Fig [4(a,b,c)] showed the change of the cross section values with the change of the central well depth values for incident energy $(5$ $\mathrm{MeV})$.
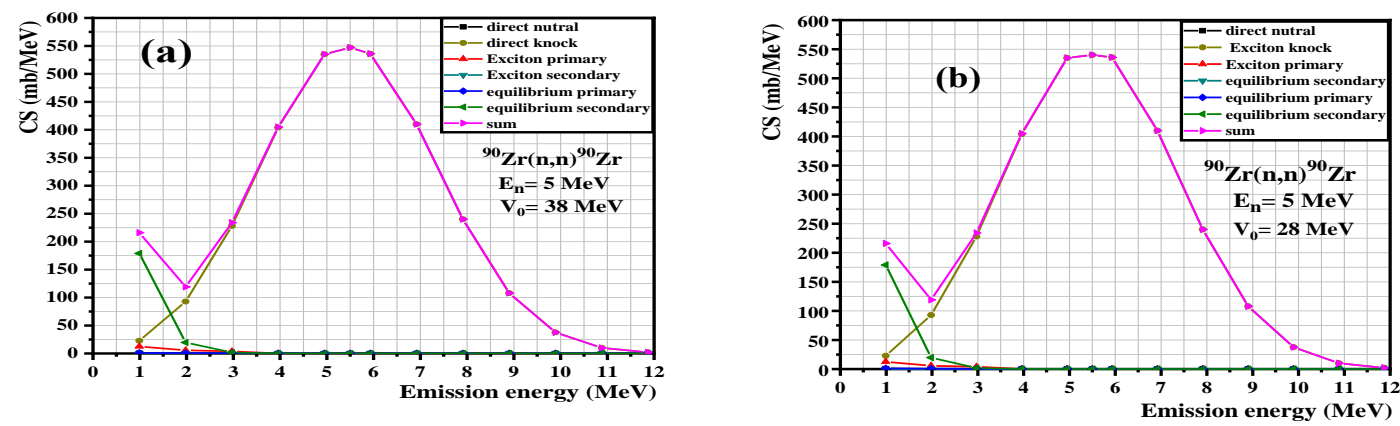


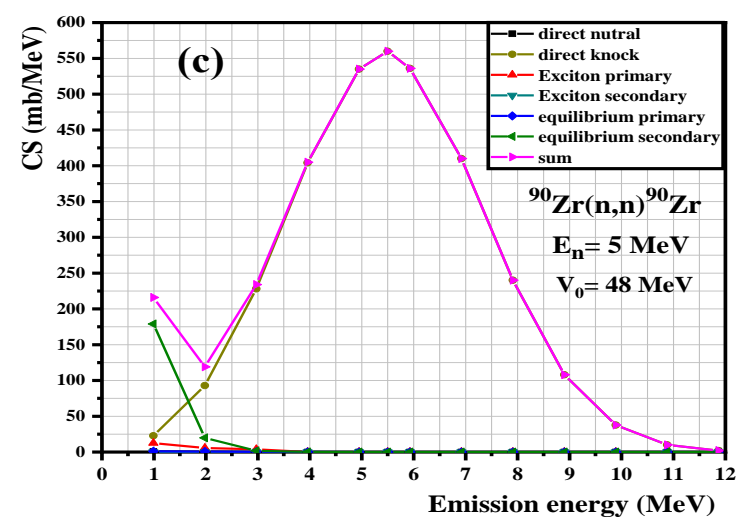

Fig.4: (a,b,c). Effect of changes the central well depth on the cross section values for the nuclear reaction $\left[{ }^{90} \mathrm{Zr}(\mathrm{n}, \mathrm{n}){ }^{90} \mathrm{Zr}\right]$

\section{References}

A. .J. J. Griffin: Phys. Rev. Lett., 17, 478 (1966); Phys. Lett., 24 B, 5 (1967).

B. J.R. Grover,and J. Gilat, , Phys. Rev. 157(1967)802.

C. J.Bisplinghoff, J , Ernst, R. Lohr, T. Mayer-Kuckuk, P. Meyer, Nucl. Phys. A 269 (1976)147.

D. C. Kalbach, Phys. Rev. C 23 (1981) 124.

E. C. Kalbach; User's Manual for PRECO-2006, Exciton Model Pre-equilibrium Nuclear Reaction Code with Direct Reactions, Triangle Universities Nuclear Laboratory, Duke University(2007).

F. H. Feshbach, A. Kerman, S. Koonin, Ann. Phys. (N.Y.) 125, 429 (1980) 476.

G. C.Kalbach ;User's manual for PRECO-2000. Duke University.2001. Retrieved from www.nndc.bnl.gov/nndcscr/model-codes/preco- 2000/.

H. C.K. Cline, and M. Blann, The pre-equilibrium statistical model: Description of the nuclear equilibration process and parameterization of the model. Nucl. Phys., A172, pp:225-259(1971).

I. C. Kalbach, Systematics of Continuum Angualr Distributions: Extensions to Higher Energies. Phys. Rev. C 37, pp:2350-2370. Kalbach, C., 1986, Two-component exciton model: basic formalism away from shell closures. Phys. Rev., C33, pp: 818-833(1988).

J. C. Kalbach, Pre-equilibrium reactions with complex particle channels, Phys. Rev. C 71, 034606, C. Kalbach, Acta Phys. Slov., 45, p:685(2005).

K. C. Kalbch, Phys. Rev. C 73(2006) 024614.

L. Shafik S. Shafik, Hadi D. Alattabi, and Masar A. Kadhim; Investigation of Isospin Conservation Effects on Pre- Equilibrium Nuclear Reactions for Some Nuclei; IOP Conf. Series: Journal of Physics: Conf. Series 1178 (2019) 012005.

M. J. P. Meulders, A.Koning, S. Leray, High and Intermediate energy Nuclear Data for Accelerator-driven Systems. HINDAS (2005).

N. A. J. Koning, S. Hilaire, S. Goriely, Talys User Manual, A nuclear reaction program,Nuclear Research and Consultancy Group (NRG) Westerduinweg, NL-1755 ZG, Petten, The Netherlands, TENDL, (2014).

O. R. Bonetti, A. J. Koning, J. M. Akkermans, P. E. Hodgson, Phys. Rep. 247 (1), 1 (1994) 58.

P. R. Bonetti, M. B. Chadwick, P. E. Hodgson, B.V. Carlson, M.S. Hussein, Phys. Rep.202, 4, 171 (1991) 231.

Q. ENDF/B-VII. 1 (Evaluated Nuclear Data File Version B) LANL, ORNL.

R. JEFF library (Joint Evaluated Fission File and Fusion File), "The JEFF-3.2 Nuclear Data Library".

S. A. Takahashi, E. Ichimura, Y. Nuclear Reaction Data", H. Osaka Univ., OKTAVIAN Reports 87 (1987) 03.

T. S. Benck, I. Slypen, J. P. Meulders, V. Corcalciuc, M. B. Chadwick, P. G. Young, A. J. Koning, "Experimental Nuclear Reaction Data" Phys. Rev. C. 58, 3 (1998) 1558.

U. Shafik S. Shafik and A. D. Salloum; Investigation of the Appropriate Partial Level Density Formula for Pre-Equilibrium Nuclear Exciton Model; Journal of Applied Mathematics and Physics, (2013), 1, 47-54.

V. J. A. Jungerman, F. P. Brady, W. J. Knox, T. Montgomery, M. R. Mcgie, J. L. Romero, Y. Ishizaki, J, NIM. 94 (1971) 421. 